\title{
Anwendernutzen auch für KMU
}

\author{
Spätestens seit Inkrafttreten der novellierten EG-Öko-Audit-Verordnung wird \\ immer deutlicher gefordert: die Umweltleistung einer Organisation muss jährlich \\ messbar kontinuierlich verbessert werden. Ein Instrument ist die ISO Norm zur \\ Umweltleistungsbewertung. Hält es in der KMU-Praxis das was es verspricht?
}

M

Von S. Richardsen und B. Grabl it der 1999 veröffentlichten Norm DIN EN ISO 14031 „Umweltleistungsbewertung“ (1) wurde Unternehmen ein Instrument an die Hand gegeben, ihre Umweltleistung systematisch und mit tragfähigen Daten zu dokumentieren.

Kann die ISO 14031 einen Beitrag zur kontinuierlichen Verbesserung der Umweltleistung leisten? Eignet sie sich auch für klein- und mittelständische Unternehmen (KMU)? Welchen Anwendernutzen sehen die KMU in der ISO 14031? Diesen Fragen wurde in einem Pilotprojekt der Fachhochschule Lübeck (2) nachgegangen.

Dazu wurden bewusst eine Branche mit Erfahrungen auf den Gebiet des Umweltmanagements (Druckerei) und eine noch wenig analysierte Branche, die Milchwirtschaft, gewählt. Als Praxispartner beteiligten sich der Norddeutsche Genossenschaftsverband und die Unternehmen Frank Druck, Preetz, Meiereigenossenschaft Holtsee, Meierei Barmstedt und Trave-Milch Leezen.

\section{Kernelemente der ISO 14031}

Die ISO 14031 beschreibt die Einfuihrung von Umweltkennzahlensystemen auf Grundlage des so genannten PDCA-Kreises: plan, do, check and act. Sie unterscheidet zwei Kennzahlenarten:

- Umweltleistungskennzahlen befassen sich direkt mit der Organisation. Sie werden nochmals unterteilt in Managementleistungskennzablen, die Informationen über die Aktivitäten des Managements hinsichtlich der Umweltleistung des Unternehmens zur Verfügung stellen und operative Leistungskennzablen, die Informationen über die Umweltleistung im operativen Bereich des Unternehmens zur Verfügung stellen, sich also mit den Stoff- und Energieströmen befassen.

- Umweltzustandskennzablen stellen Informationen über die Organisationsumwelt dar.

Die Norm betont, dass die Umweltleistungsbewertung ein fortlaufender interner Managementprozess zur Auswahl, Erfassung und Auswertung von
Daten und Informationen ist. Ziele sind die Optimierung der Informationsbasis zur Bewertung der Umweltleistung, die Darstellung und Kommunikation von Leistungstrends sowie die Verbesserung der Umweltleistung.

\section{Unklarheiten für die Umsetzung}

In dem Projekt wurde analog zur ISO 14031 eine Methodik entwickelt, Umweltkennzahlensysteme praxisnah und maßgeschneidert für das jeweilige Unternehmen zu entwickeln und zu implementieren. Dabei haben sich folgende Unklarheiten der Norm herauskristallisiert:

Zielfindung: Im Arbeitsschritt planen wird der Ansatz verfolgt, eine Kennzahlenliste unter Berücksichtigung der interessierten Kreise, der wesentlichen Umweltaspekte und der Umweltleistungskriterien zu erarbeiten. Die Berücksichtigung der interessierten Kreise zeigte sich als sehr nützlich und

wandlungen der Norm empfehlen wir eine Harmonisierung oder klare Abgrenzung der Begriffe Öko-

ist in der Norm mit anschaulichen Beispielen hinterlegt. Zu den anderen Kriterien wird in der Norm wenig Hilfestellung gegeben.

Verbesserung von Umweltleistung und Umweltleistungsbewertung: Die Norm zielt sowohl auf die Verbesserung der Umweltleistungsbewertung als auch der Umweltleistung. Diese beiden Aspekte sind allerdings nicht systematisch behandelt. Eine vereinfachte Terminologie und klare unmissverständliche Anleitungen, wie beide Zielsetzungen in der Organisation entwickelt werden können, wären hier wünschenswert.

Prïfen und Handeln: Die Norm beschreibt in drei Schritten den Managementkreislauf planen - umsetzen - prüfen/handeln. Dabei ist der Arbeitsschritt umsetzen nochmals in vier Unterarbeitsschritte gegliedert: Datenerfassung, Datenanalyse und -aufbereitung, Informationsbeurteilung, Berichterstattung und Kommunikation. In der Informationsbeurteilung werden bereits Maßnahmen zur Unterstuitzung der Verbesserung der Umweltleistung angesprochen - eigentlich ein Arbeitsschritt, der unter prüfen/ handeln zu erwarten wäre. Auch die Berichterstattung und Kommunikation lässt sich eher dem Arbeitsschritt prüfen/handeln zuordnen. Die in der Norm vorgeschlagene Struktur erwies sich für die Praxispartner als zu verschachtelt.

Umweltleistungsbewertung und ökocontrolling: Neben den oben genannten strukturellen Ab-

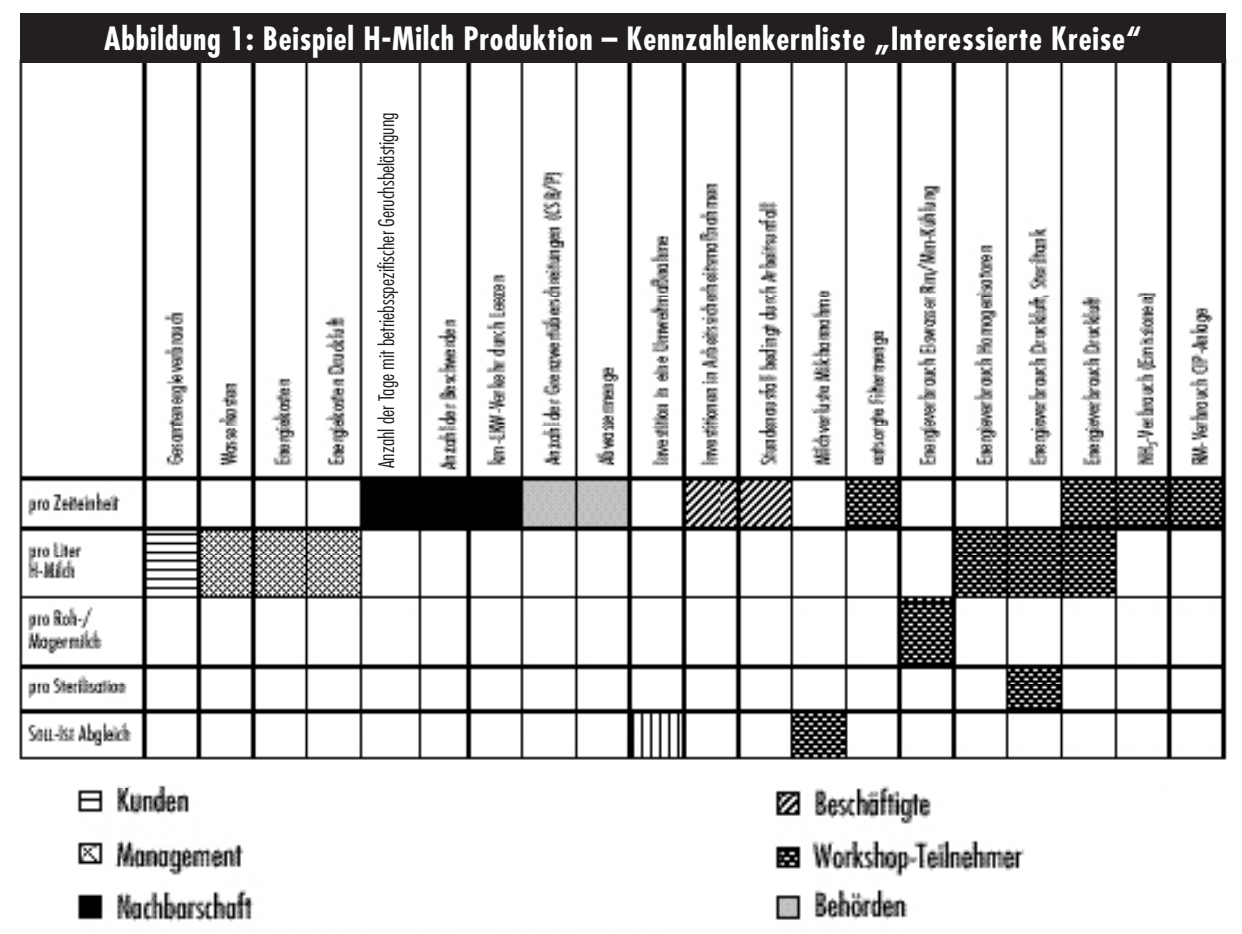

ए Lieferonfen/Gesellschafter (identisch, da Genossenschaft) Quelle: eigene Darstellung 
controlling/Umweltcontrolling und Umweltleistungsbewertung. In der Literatur wird häufig der Eindruck vermittelt, als handele es sich um zwei völlig unterschiedliche Methoden, was für interessierte Organisationen verwirrend ist. Ein derzeit laufendes Projekt beschäftigt sich mit dieser Problematik (3).

\section{Umsetzungsmethodik}

Der in der ISO 14031 zugrunde gelegte PDCAKreis wurde in dem Pilotprojekt folgendermaßen umgesetzt:

Bei der Planung der Umweltkennzablen wurden zunächst weder das vorhandene Datenmaterial noch die Handlungsspielräume berücksichtigt. Allein die Anforderungen der interessierten Kreise und die betrieblichen Umweltaspekte entlang der Prozesslandkarte waren entscheidend. Auf diese Weise erhielten die Unternehmen eine betriebsspezifische maßgeschneiderte „Kennzahlenwunschliste“.

Durch diese Vorgehensweise

- wurde gezielt erfasst, welche Informationen für die jeweiligen interessierten Kreise wichtig sind (Beispiele in Abb. 1 auf S. 29).

- konnten die wesentlichen Umweltaspekte praxisnah entlang der betrieblichen Prozesse ermittelt werden (Beispiele in Abb. 2).

Erst in der Umsetzungsphase, während der Datenerfassung, Datenanalyse und -aufbereitung wurden die Handlungsspielräume der Unternehmen berücksichtigt. In einer Aufwand-NutzenAnalyse wurde geprüft, welche Kennzahlen der

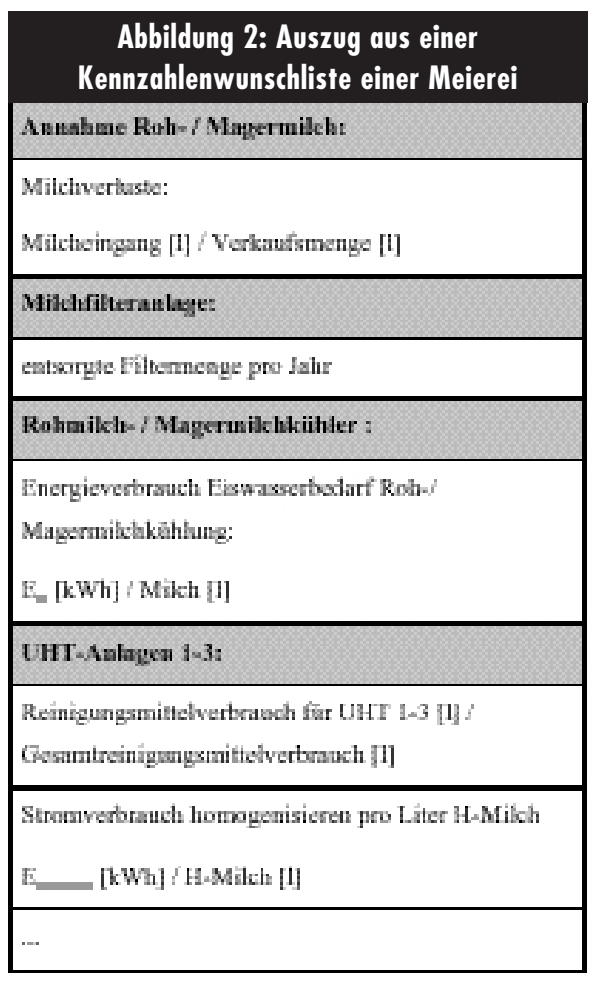

Quelle: eigene Darstellung umfangreichen Wunschliste in diesem ersten Durchlauf des PDCA-Kreises in die Praxis umgesetzt werden.

Da es sich in den beteiligten Unternehmen um eine erste systematische Bestandsaufnahme von Kennzahlen zur Umweltleistungsbewertung nach ISO 14031 handelte, wurden in dem Arbeitsschritt prïfen und handeln

- die erhobenen Kennzahlen auf Brauchbarkeit hin überprüft,

- Vorschläge entwickelt, die Datenerfassung, Datendokumentation und Kennzahlenbildung in die üblichen Betriebsabläufe einzubinden und

- für erkannte Schwachstellen mit der Maßnahmenplanung begonnen.

Im Rahmen des Projektes wurden die beteiligten Unternehmen einmal durch den Prozess der Umweltleistungsbewertung begleitet. Hierbei zeigte sich, dass der Schwerpunkt zunächst in der Verbesserung der Umweltleistungsbewertung durch Verbesserung der Informations- und Datenlage liegt. Nach und nach wird eine Verschiebung des Schwerpunktes in Richtung Verbesserung der Umweltleistung durch operative und organisatorische Maßnahmen erwartet.

\section{Wesentliche Ergebnisse}

Auf betrieblicher Ebene wurden folgende Ziele erreicht:

- Durch die Berücksichtigung interessierter Kreise als Zielgruppe interner und externer Kommunikation konnte eine marktorientierte Planung von Umweltkennzahlen durchgeführt werden.

- Die Verbindung zum Umweltkostenmanagement wurde transparent gemacht.

- Der Anwendernutzen von Umweltkennzahlen wurde vermittelt, zum Beispiel zur Prüfung und Vorbereitung investiver Maßnahmen.

- Die Vorteile einer prozessorientierten Einführung von Umweltmanagementsystemen wurden verdeutlicht.

- Es wurde ein Verfahren entwickelt, um mit wenig externem Beratungsaufwand maßgeschneiderte Kennzahlensysteme für KMU-Bedürfnisse zu erarbeiten.

Mit Hilfe des implementierten Umweltkennzahlensystems haben die Unternehmen die Möglichkeit erhalten, ihre

- Umweltziele kontinuierlich zu überprüfen und zu steuern und deren Umsetzung mit tragfähigen Daten zu dokumentieren.

- Optimierungspotentiale aufzudecken und mit Daten zu hinterlegen.
Umweltleistung intern als auch extern zu kommunizieren.

Es hat sich gezeigt, dass die ISO 14031 sowohl für Unternehmen mit formalem Umweltmanagementsystem, als auch für Unternehmen ohne Umweltmanagementsystem ein geeignetes Werkzeug darstellt. In allen beteiligten Unternehmen wurden bislang hauptsächlich die Stoff- und Energieströme für den gesamten Betrieb erfasst. Prozessbezogene Daten, Daten für die einzelnen Abteilungen, Daten die den jeweiligen Arbeitsschichten zugeordnet werden können, fehlten bei Projektbeginn weitgehend.

Dem nach EMAS zertifizierten Unternehmen wurde erst durch die Einführung des Umweltkennzahlensystems ermöglicht, das Umweltprogramm mit tragfähigen Daten zu hinterlegen und zu überprüfen. In den Unternehmen ohne Umweltmanagementsystem konnten sowohl allgemeine Umweltziele mit Daten hinterlegt als auch neue Ansätze zur Verbesserung der Umweltleistung herausgearbeitet werden, zum Beispiel prozessbezogene Schwerpunkte des Energieverbrauchs oder die Optimierung der Produktpalette an Reinigungsmitteln. Durch das Aufzeigen der für das jeweilige Unternehmen relevanten Umweltaspekte und der systematisierten Datenerhebung und -pflege fühlten sich zwei der beteiligten Meiereien zudem gut vorbereitet für die Einführung eines Umweltmanagementsystems.

\section{Anmerkungen}

(1) DIN EN ISO 14031. Umweltmanagement - Umweltleistungsbewertung - Leitlinien. Beuth Verlag, Berlin 2000.

(2) Richardsen, S./ Grahl, B.: Umweltleistungsbewertung nach DIN EN ISO 14031. Anwendernutzen von Umweltkennzahlen für kleinere und mittlere Unternehmen; Studie gefördert vom Ministerium für Umwelt, Natur und Forsten Schleswig-Holstein. Fachhochschule Lübeck, 2001.

(3) Loew, T./ Beucker, S./ Jürgens, G.: Vergleichende Analyse der Umweltcontrollinginstrumente Umweltbilanz, Umweltkennzahlen und Flusskostenrechnung. Diskussionspapier des IÖW. Berlin 2002.

\section{Die Autorinnen}

Susanne Richardsen ist Mitarbeiterin des Otto Versands.

Kontakt: Otto Versand, Umwelt- und Gesellschaftspolitik, Wandsbeker Str. 3-7, 22179 Hamburg. Tel. 040/ 6461-1375,

E-Mail: susanne.richardsen@otto.de

Dr. Birgit Grahl ist Professorin an der Fachhochschule Lübeck.

Kontakt: FH Lübeck, FB Angewandte Naturwissenschaften, Stephensonstr. 3, 23562 lübeck.

E-Mail: grahl@fh-luebeck.de 
(c) 20I0 Authors; licensee IÖW and oekom verlag. This is an article distributed under the terms of the Creative Commons Attribution Non-Commercial No Derivates License (http://creativecommons.org/licenses/by-nc-nd/3.o/), which permits unrestricted use, distribution, and reproduction in any medium, provided the original work is properly cited. 